\title{
Transmission-Constrained Residual Demand Derivative in Electricity Markets
}

\author{
Lin Xu, Student Member, IEEE, and Ross Baldick, Fellow, IEEE
}

\begin{abstract}
The residual demand derivative plays a central role in constructing the best response to competitors' strategies in widely used strategic models such as the Cournot model and the supply function model. In the absence of transportation or transmission constraints, the residual demand derivative is obtained straightforwardly by taking the derivative of the residual demand function with respect to price. However, in an electricity market, the market is embedded in a transmission network. When there is no transmission congestion, the residual demand derivative can be calculated as usual, but when there is transmission congestion, the residual demand derivative is more difficult to calculate. In this paper, we characterize the transmission-constrained residual demand derivative. We use the dc power flow model and characterize the residual demand derivative analytically. The residual demand derivative could also be obtained from the solution of a specific weighted least squares problem. Several properties of the residual demand derivative are implications of the weighted least squares theory. We also characterize the condition under which the residual demand derivative will be bounded or unbounded when there are perfectly elastic supplies/demands at some buses in the system. We verified our results in three examples: a two-bus system, a four-bus two-loop system, and a three-bus one-loop system with one perfectly elastic supply. The residual demand derivative characterization can be used to analyze the strategic behavior in both the Cournot model and the supply function model with transmission constraints, and it can be easily incorporated into sophisticated optimal strategy algorithms.
\end{abstract}

Index Terms-Best response, Cournot model, electricity market, residual demand, supply function equilibrium, transmission constraint.

\section{INTRODUCTION}

$\mathbf{E}$ LECTRICITY markets have been widely studied by economists, engineers, and policy makers. One of the most important issues comes from the special nature of electricity transmission networks [1]. Although numerical case studies can provide insights into the impacts of binding transmission constraints [1]-[5], analytical results can be more valuable and informative. In this paper, we will analytically characterize the residual demand derivative when there are binding transmission constraints.

Our main focus in this paper is an offer-based electricity energy market, which is cleared by a security-constrained central economic dispatch and pricing mechanism [6]. Following [2]-[5], we assume dc optimal power flow (OPF) and nodal

Manuscript received January 29, 2007; revised June 18, 2007. This work was supported in part by NSF Grant ECS0422914 and in part by PSERC. Paper no. TPWRS-00035-2007.

The authors are with the University of Texas at Austin, Austin, TX 78712 USA (e-mail: linxu@mail.utexas.edu; baldick@ece.utexas.edu).

Digital Object Identifier 10.1109/TPWRS.2007.907511 pricing as the central dispatch and pricing mechanism in this paper.

The supply function model is widely used to model the competition in offer-based electricity markets. A generation firm is assumed to submit a supply function specifying its maximum power at each price level (or equivalently submit the inverse of its supply function). The inputs to the OPF are the inverse functions of the supply functions, and the outputs are the market clearing prices and quantities.

The transmission-unconstrained best response in a supply function model is characterized in [7]-[10]. We rewrite the characterization as

$$
S_{i}(p)=-\left(p-C_{i}^{\prime}\left(S_{i}(p)\right)\right) R_{i}^{\prime}(p)
$$

where

- $p$ is the market price;

- $C_{i}^{\prime}(\bullet)$ is player $i$ 's marginal cost function;

- $S_{i}(\bullet)$ is player $i$ 's supply function;

- $R_{i}^{\prime}(\bullet)$ is player $i$ 's residual demand derivative function;

- player $i$ 's residual demand function, $R_{i}(\bullet)$, is defined by

$$
R_{i}(p)=D(p)-S_{-i}(p), \forall p \in\left[p_{\min }, p_{\max }\right]
$$

where

- $S_{-i}(p)$ is defined by

$$
S_{-i}(p)=\sum_{j \neq i} S_{j}(p), \forall p \in\left[p_{\min }, p_{\max }\right] ;
$$

- $D(\bullet)$ is the total demand function of the market;

- $\left[p_{\min }, p_{\max }\right]$ is the range of possible market prices.

As specified in (2), a generation firm's residual demand is defined as the demand minus the aggregated supply of all other suppliers in the market. Typically, the residual demand function has negative slope, reflecting the fact that, as price increases, demand decreases while supply increases.

The derivative of the residual demand is useful for market participants, analysts, and market monitoring entities. For generation firms, an individual generation firm's optimal supply function depends on its residual demand derivative $R_{i}^{\prime}(p)$ as characterized in (1), so the residual demand derivative plays a central role in constructing optimal bidding strategies. If the residual demand derivative is available, the best response strategy can be constructed based on (1). For market analysts using strategic equilibrium models, such as the Cournot model and the supply function equilibrium (SFE) model, the residual demand derivative is crucial for characterizing the market Nash equilibrium. For market monitoring entities, the residual demand derivative 
implicitly indicates the potential market power a player could possibly exert. The more elastic the residual demand curve is, the less influence a player has on price, and the less is its market power potential.

In generic markets without transportation or transmission constraints, the residual demand derivative is obtained easily by taking the derivative of the right-hand side of (2) with respect to price $p$. The electricity market is unique in the sense that it is embedded in a transmission network. The unique feature is that the transmission network has transmission capacity limits and thus is possibly congested. Transmission congestion leads to difficulty in computing the residual demand derivative.

We will derive the transmission-constrained residual demand derivative based on the supply function model. The SFE has been widely used to study strategic behaviors and market power in electricity markets. It was introduced by Klemperer and Meyer in [7]. Considerable further developments and applications have focused on electricity markets [5], [8]-[18].

To the best of the authors' knowledge, no previous paper has presented the calculation of the transmission-constrained residual demand derivative and provided the analytic solution as we do in this paper. Most papers calculate the best response numerically from the profit maximization problem instead of calculating the residual demand derivative and then utilizing (1) to obtain the best response. For example, Cardell et al. use a Cournot model in a three-bus looped network and numerically demonstrate that strategic behaviors involving the transmission constraints could lead to a market outcome that is different from the usual analysis of imperfect competition [1]. Their work reinforces the need for more rigorous characterization of the effect of the transmission constraints on residual demand. Although we make derivations based on the supply function model in this paper, similar analysis also applies to the Cournot model. As an example, we will demonstrate how to verify Cardell et al.'s Cournot model three-bus looped network computational results in [1].

Yao and Oren propose an equilibrium programming with equilibrium constraints (EPEC) model to calculate the market equilibrium in a transmission-constrained network [2]. However, their characterization of the transmission constraint is partial in the sense that they assume the residual demand functions are explicitly given and do not change as transmission congestion conditions change. As will be shown in this paper, the residual demand function will be different if the binding transmission constraints change, and the calculation of the residual demand derivative given binding transmission constraints is the main contribution of this paper. Our results in this paper provide the basis for better transmission constraint modeling and could be incorporated into algorithms like Yao and Oren's to represent more accurate transmission constraint characterization, albeit possibly at the cost of making profit functions non-concave due to the change of binding constraints. In that case, we may need to enumerate all possible combinations of binding constraints in searching for the best response.

$\mathrm{Xu}$ and $\mathrm{Yu}$ use a linear supply function model and calculate the transmission-constrained SFE [5]. They consider the impact of the transmission and calculate the best response numerically. In this paper, we will characterize the transmission-con- strained residual demand derivative analytically and verify the results with reference to $\mathrm{Xu}$ and Yu's four-bus looped network example.

The approach in this paper can also be viewed as a generalization of the methods in [19] and [20]. References [19] and [20] calculate the sensitivities of the generation dispatches to offer prices considering the transmission constraints. Our approach generalizes their methods in that we can handle any type of offer functions and not just fixed price offers. Our emphasis is on the residual demand, whose derivative reveals the sensitivities of the generation dispatch to the incremental market price changes. We will demonstrate that these sensitivities are independent of the offer functional forms but do depend on the binding generation capacity and transmission constraints. We can handle all these constraints without any difficulty, whereas the methods in [19] and [20] cannot directly handle the binding generation capacity constraints.

The organization of the rest of this paper is as follows. Section II presents the analytic calculation of the residual demand derivative in a transmission-constrained network and its properties, Section III provides one intuitive example and two numerical examples taken from previous papers to verify our calculation, and Section IV concludes. In addition, the Appendix provides background knowledge about ordinary least squares (OLS) problem and weighted least squares (WLS) problem.

\section{RESIDUAL DEMAND DERIVATIVE}

In a typical electricity market, let us assume that different generation firms are located at different buses, and let us index the firms by the bus number. Under nodal pricing, transmission constraints will generally lead to different nodal prices for different buses, so instead of having only one uniform market price $p$, we will have a vector of nodal equilibrium prices

$$
\mathbf{p}=\left[\begin{array}{llll}
p_{1} & p_{2} & \cdots & p_{n}
\end{array}\right]^{T}
$$

assuming there are $n$ buses in the system.

We assume that the demand at each bus depends on only its local price, because in the short term, it is unlikely that a market participant could shift loads between buses according to real-time nodal prices. Currently, the real-time prices are not published quickly enough to support this kind of load response in electricity markets.

Accordingly, a generation firm's residual demand will be a function of its local nodal price. Write out the energy balance condition

$$
\sum_{j=1}^{n}\left(D_{j}\left(p_{j}\right)-S_{j}\left(p_{j}\right)\right)=0
$$

By keeping a specific $S_{i}\left(p_{i}\right)-D_{i}\left(p_{i}\right)$ on the left-hand side, and moving all other terms to the right-hand side, we obtain

$$
S_{i}\left(p_{i}\right)-D_{i}\left(p_{i}\right)=\sum_{j=1, j \neq i}^{n}\left(D_{j}\left(p_{j}\right)-S_{j}\left(p_{j}\right)\right) .
$$

Before we continue, to simplify notation, we combine each generation firm's supply function with the demand curve at 
the same bus by treating demand as negative supply, and this process will result in only one supply function left at each bus so that (5) becomes

$$
S_{i}\left(p_{i}\right)=\sum_{j=1, j \neq i}^{n}-S_{j}\left(p_{j}\right) .
$$

The market clearing condition for the residual market at bus $i$ implies that the residual demand equals supply, i.e.,

$$
R_{i}\left(p_{i}\right)=S_{i}\left(p_{i}\right) .
$$

Now we are going to derive $R_{i}^{\prime}\left(p_{i}\right)$ analytically. Without loss of generality, we want to calculate $R_{i}^{\prime}\left(p_{i}\right)$ at a specific bus $i$. We choose this bus to be the slack bus, and we reorder all the system buses to number this chosen bus to be bus $n$. That is, we want to calculate the residual demand derivative $R_{n}^{\prime}\left(p_{n}\right)$ at the slack bus $n$. The corresponding offer cost function, whose derivative is the inverse of the supply function $S_{i}(\bullet)$, is denoted by $O_{i}(\bullet)$. That is, we define $O_{i}(\bullet)$ by

$$
\forall p_{i}, \quad p_{i}=O_{i}^{\prime}\left(S_{i}\left(p_{i}\right)\right)
$$

Also we assume the functions $O_{i}(\bullet), \forall i=1, \ldots, n$ are strictly convex and twice partially differentiable.

Following [21], we assume the market is cleared by solving the following dc OPF problem:

$$
\begin{aligned}
& \min _{\mathbf{q}} \sum_{i=1}^{n} O_{i}\left(q_{i}\right) \\
& \text { s.t. } \mathbf{H q} \leq \mathbf{Z} \\
& q_{n}^{\min } \leq q_{n} \leq q_{n}^{\max } \\
& \mathbf{1}^{T} \mathbf{q}=\mathbf{0}
\end{aligned}
$$

where

- bus $n$ is the slack bus;

- $\mathbf{q}=\left[\begin{array}{llll}q_{1} & q_{2} & \ldots & q_{n}\end{array}\right]^{T}$ is the nodal power injection quantity vector;

- equation (10) consists of the transmission constraints and the generation capacity constraints for non-slack buses (suppose there are totally $m$ of them);

- $\mathbf{H}$ is a $m \times n$ matrix consisting of the submatrix of power transfer distribution factors (PTDFs) corresponding to the transmission constraints and the submatrix representing the capacity constraints for non-slack buses;

- $\mathbf{Z}$ consists of the transmission capacity limits and the generation capacity limits for non-slack buses;

- $\mathbf{1}=\underbrace{\left[\begin{array}{llll}1 & 1 & \ldots & 1\end{array}\right]^{T}}_{n}$;

- equation (11) ${ }^{n}$ is the generation capacity constraint, which specifies the upper and lower limits of the domain of the offer cost function at the slack bus;

- equation (12) is the energy balance constraint.

There are two widely used OPF formulations. One is to consider elastic demands, and the OPF objective is to maximize total social welfare; the other is to consider inelastic demand, and the OPF objective is to minimize total generation cost. We use the first OPF formulation in this paper to derive the residual demand derivative. However, we stress that the methodology is also applicable to the alternate OPF formulation.
We intend to calculate the residual demand derivative evaluated at the current market operating point. The current market operating point is determined by the OPF solution. Therefore, the residual demand derivative calculation is a post-OPF analysis. Given an OPF solution, we know which constraints are binding in the OPF formulation. Given these binding OPF constraints at the solution, we will form the Lagrangian for the OPF problem (9)-(12) including only binding constraints. Let us denote the binding constraints by subscript " $b$ ". The calculation needs to be separated into two cases:

- the slack bus generation capacity constraint (11) is not binding;

- the slack bus generation capacity constraint (11) is binding, but we will see that the two cases result in the same expression for the residual demand derivative.

\section{A. Non-Binding Slack Bus Generation Capacity Constraint}

The Lagrangian for (9)-(12) including only the binding constraints is as follows:

$$
\mathcal{L}=\sum_{i=1}^{n} O_{i}\left(q_{i}\right)-\lambda \sum_{i=1}^{n} q_{i}+\boldsymbol{\mu}_{b}^{T}\left(\mathbf{H}_{b} \mathbf{q}-\mathbf{Z}_{b}\right) .
$$

The first-order necessary conditions (FONCs) of (13a) are

$$
\begin{aligned}
O_{i}^{\prime}\left(q_{i}\right) & =\lambda+\boldsymbol{\mu}_{b}^{T} \overline{\mathbf{H}}_{b i}, i=1, \ldots, n-1 \\
O_{n}^{\prime}\left(q_{n}\right) & =\lambda \\
\overline{\mathbf{H}}_{b} \overline{\mathbf{q}} & =\mathbf{Z}_{b} \\
q_{n} & =-\sum_{i=1}^{n-1} q_{i}
\end{aligned}
$$

where

- $\overline{\mathbf{H}}_{b}$ is a $m_{b} \times(n-1)$ matrix generated by eliminating the $n$th column (all elements in this column are zeros) of $\mathbf{H}_{b}$;

- $\overline{\mathbf{H}}_{b i}$ is the $i$ th column of $\mathbf{H}_{b}$;

- $\overline{\mathbf{q}}$ is obtained from q by eliminating the $n$th entry;

- $\mathbf{Z}_{b}$ consists of the binding transmission capacity limits and the binding generation capacity limits for non-slack buses.

Because the generation capacity constraint is not binding at bus $n$, the marginal offer cost at bus $n$ is equal to the market clearing nodal price at bus $n$ given by the Lagrange multiplier $\lambda$ according to (15a). There are $n+m_{b}+1$ equations, and $n+m_{b}+1$ variables in the FONCs (14a)-(17a), so the FONCs are uniquely solvable assuming regularity conditions. Denote the solution by

$$
\left[\begin{array}{lllllll}
\hat{q}_{1} & \ldots & \hat{q}_{n} & \hat{\lambda} & \hat{\mu}_{1} & \ldots & \hat{\mu}_{m_{b}}
\end{array}\right]^{T} .
$$

The FONCs characterize a market clearing point. From the perspective of the generation firm located at the slack bus, the market clearing point $\left(\hat{\lambda}, \hat{q}_{n}\right)$ is the intersection of its own offer cost function and the residual demand function. If the generation firm located at the slack bus changes its offer function, the market will clear at a different point, which is the new intersection point of the changed offer function and the residual demand function. In other words, the market clearing points generated by changing the offer function of the generation firm located at the slack bus are all points on the residual demand function. Therefore, the residual demand function is characterized by the locus 
of the market clearing points $\left(\lambda, q_{n}\right)$ obtained by changing the generation firm's offer function.

That is, if we remove the equations that contain the offer information of the generation firm located at the slack bus from the FONCs, the remaining equations characterize the residual demand function, because the residual demand function should not depend on a generation firm's own offer information. In the FONCs (14a)-(17a), (15a) contains $O_{n}^{\prime}(\bullet)$, which is based on the offer cost function of the generation firm located at the slack bus, so we should remove it from the FONCs to characterize the residual demand function. With (15a) removed, we have $n+m_{b}$ equations, namely, (14a), (16a), and (17a), and $n+m_{b}+1$ variables left in the FONCs, so there is one degree of freedom. The one degree of freedom implicitly characterizes a locus of $\left(\lambda, q_{n}\right)$, i.e., the residual demand curve.

\section{B. Binding Slack Bus Generation Capacity Constraint}

Consider that the upper generation capacity constraint is binding at the slack bus. The Lagrangian for (9)-(12) including only the binding constraints is as follows:

$$
\begin{aligned}
& \mathcal{L}=\sum_{i=1}^{n} O_{i}\left(q_{i}\right)-\lambda \sum_{i=1}^{n} q_{i}+\boldsymbol{\mu}_{b}^{T}\left(\mathbf{H}_{b} \mathbf{q}-\mathbf{Z}_{b}\right) \\
&+\rho^{\max }\left(q_{n}-q_{n}^{\max }\right)
\end{aligned}
$$

where, without loss of generality, we have assumed that the maximum generation capacity constraint is binding. A similar analysis applies for the minimum generation capacity constraint. The FONCs of (13b) are

$$
\begin{aligned}
O_{i}^{\prime}\left(q_{i}\right) & =\lambda+\boldsymbol{\mu}_{b}^{T} \overline{\mathbf{H}}_{b i}, i=1, \ldots, n-1 \\
O_{n}^{\prime}\left(q_{n}\right)-\rho^{\max } & =\lambda \\
\overline{\mathbf{H}}_{b} \overline{\mathbf{q}} & =\mathbf{Z}_{b} \\
q_{n} & =-\sum_{i=1}^{n-1} q_{i} \\
q_{n} & =q_{n}^{\max } .
\end{aligned}
$$

Denote the solution of (14b)-(18b) by

$$
\left[\begin{array}{llllllll}
\hat{q}_{1} & \ldots & \hat{q}_{n} & \hat{\lambda} & \hat{\mu}_{1} & \ldots & \hat{\mu}_{m} & \hat{\rho}^{\max }
\end{array}\right]^{T} .
$$

We need to clarify that generally speaking, we could not choose a bus with binding generation capacity constraint as the slack bus to solve problems involving power flow. The reason why we can do this in this case is that the OPF is already solved, so in this post-OPF analysis, from the optimality conditions point of view, it does not matter which bus is the slack bus as long as the FONCs (14b)-(18b) are satisfied.

For the same reason as in Section II-A, we need to remove the equations that contain the offer information of the generation firm located at the slack bus from the FONCs (14b)-(18b). Again, (15b) contains $O_{n}^{\prime}(\bullet)$ and thus should be removed. In this case, in addition, another equation, (18b), should also be removed, because it specifies the upper limit of the offer function domain, and thus, $O_{n}(\bullet)$ and (18b) together characterize the offer information.

Note that if (15b) and (18b) are removed, the remaining (14b), (16b), and (17b) are exactly the same as (14a), (16a), and (17a).

\section{Sensitivity Analysis}

Now we are going to calculate the residual demand derivative at bus $n$ by simultaneously solving (14a), (16a), and (17a), which has one degree of freedom that characterizes a locus of $\left(\lambda, q_{n}\right)$.

Consider (14a), (16a), and (17a) parameterized by $\lambda$. By the Implicit Function Theorem, if second-order sufficient conditions hold, then a unique function

$$
\left[\begin{array}{llllll}
\tilde{q}_{1} & \ldots & \tilde{q}_{n} & \tilde{\mu}_{1} & \ldots & \tilde{\mu}_{m}
\end{array}\right]^{T}(\lambda)
$$

exists in a neighborhood of $\hat{\lambda}$ that solves (14a), (16a), and (17a). Furthermore

$$
\left[\tilde{q}_{1} \ldots \tilde{q}_{n} \tilde{\mu}_{1} \ldots \tilde{\mu}_{m}\right]^{T}(\hat{\lambda})=\left[\hat{q}_{1} \ldots \hat{q}_{n} \hat{\mu}_{1} \ldots \hat{\mu}_{m}\right]^{T} .
$$

Because the equations, which contain the offer information at the slack bus, have been removed, the quantity $q_{n}$ in (17a) is actually the residual demand quantity $R_{n}$, so we replace $q_{n}$ by $R_{n}$ in the left-hand side of (17a), and therefore (17a) becomes

$$
R_{n}(\lambda)=-\sum_{i=1}^{n-1} \tilde{q}_{i}(\lambda) .
$$

We are interested in the residual demand derivative, i.e., the derivative of $R_{n}(\lambda)$ with respect to $\lambda$ evaluated at $\hat{\lambda}$

$$
\frac{d R_{n}}{d \lambda}(\hat{\lambda})=-\sum_{i=1}^{n-1} \frac{d \tilde{q}_{i}(\hat{\lambda})}{d \lambda}
$$

Sensitivity analysis enables us to calculate the derivative of

$$
\left[\begin{array}{llllll}
\tilde{q}_{1} & \ldots & \tilde{q}_{n} & \tilde{\mu}_{1} & \ldots & \tilde{\mu}_{m}
\end{array}\right](\lambda)
$$

with respect to $\lambda$ evaluated at $\hat{\lambda}$. It may be that the sensitivity is not defined due to non-differentiability, and we will briefly discuss this case in Section II-E below.

From (14a) and (16a), we get

$$
\begin{aligned}
O_{i}^{\prime}\left(\tilde{q}_{i}(\lambda)\right) & =\lambda+\tilde{\boldsymbol{\mu}}_{b}^{T}(\lambda) \overline{\mathbf{H}}_{b i}, i=1, \ldots, n-1 \\
\overline{\mathbf{H}}_{b} \tilde{\overline{\mathbf{q}}}(\lambda) & =\mathbf{Z}_{b}
\end{aligned}
$$

where

$$
\tilde{\overline{\mathbf{q}}}(\lambda)=\left[\begin{array}{llll}
\tilde{q}_{1} & \tilde{q}_{2} & \ldots & \tilde{q}_{n-1}
\end{array}\right]^{T}(\lambda) .
$$

The vector $(d \tilde{\overline{\mathbf{q}}} / d \lambda)(\hat{\lambda})$ can be calculated by totally differentiating (22) and (23) with respect to $\lambda$ to obtain

$$
\left[\begin{array}{ccc}
O_{1}^{\prime \prime}\left(\hat{q}_{1}\right) & \ldots & \mathbf{0} \\
\vdots & \ddots & \vdots \\
\mathbf{0} & \ldots & O_{n-1}^{\prime \prime}\left(\hat{q}_{n-1}\right)
\end{array}\right] \frac{d \tilde{\overline{\mathbf{q}}}}{d \lambda}(\hat{\lambda})-\overline{\mathbf{H}}_{b}^{T} \frac{d \tilde{\boldsymbol{\mu}}}{d \lambda}(\hat{\lambda})=\overline{\mathbf{1}}
$$

$$
\overline{\mathbf{H}}_{b} \frac{d \tilde{\overline{\mathbf{q}}}}{d \lambda}(\hat{\lambda})=\mathbf{0}
$$


where $\overline{1}=\underbrace{\left[\begin{array}{llll}1 & 1 & \ldots & 1\end{array}\right]^{T}}_{n-1}$. Solving (24) and (25), we obtain

$$
\begin{aligned}
& \frac{d \tilde{\boldsymbol{\mu}}}{d \lambda}(\hat{\lambda})=-\left(\overline{\mathbf{H}}_{b} \boldsymbol{\Lambda} \overline{\mathbf{H}}_{b}^{T}\right)^{-1} \overline{\mathbf{H}}_{b} \boldsymbol{\Lambda} \overline{\mathbf{1}} \\
& \frac{d \tilde{\overline{\mathbf{q}}}}{d \lambda}(\hat{\lambda})=\boldsymbol{\Lambda}\left(\overline{\mathbf{I}}-\overline{\mathbf{H}}_{b}^{T}\left(\overline{\mathbf{H}}_{b} \boldsymbol{\Lambda} \overline{\mathbf{H}}_{b}^{T}\right)^{-1} \overline{\mathbf{H}}_{b} \boldsymbol{\Lambda}\right) \overline{\mathbf{1}}
\end{aligned}
$$

where

$$
\begin{aligned}
\boldsymbol{\Lambda} & =\left[\begin{array}{ccc}
O_{1}^{\prime \prime}\left(\hat{q}_{1}\right) & \ldots & \mathbf{0} \\
\vdots & \ddots & \vdots \\
\mathbf{0} & \ldots & O_{n-1}^{\prime \prime}\left(\hat{q}_{n-1}\right)
\end{array}\right]^{-1} \\
& =\left[\begin{array}{ccc}
S_{1}^{\prime}\left(\hat{p}_{1}\right) & \ldots & \mathbf{0} \\
\vdots & \ddots & \vdots \\
\mathbf{0} & \ldots & S_{n-1}^{\prime}\left(\hat{p}_{n-1}\right)
\end{array}\right]
\end{aligned}
$$

and $\overline{\mathbf{I}}$ is the $(n-1) \times(n-1)$ identity matrix. Because we assume the strict convexity of $O_{i}^{\prime \prime}(\bullet), \forall i=1, \ldots, n$, the inverse in (28) exists, and $\boldsymbol{\Lambda}$ is positive definite. Therefore

$$
\begin{aligned}
\frac{d \tilde{R}_{n}}{d \lambda}(\hat{\lambda}) & =-\overline{\mathbf{1}}^{T} \frac{d \tilde{\overline{\mathbf{q}}}}{d \lambda}(\hat{\lambda}) \\
& =-\overline{\mathbf{1}}^{T} \boldsymbol{\Lambda} \overline{\mathbf{1}}+\overline{\mathbf{1}}^{T} \boldsymbol{\Lambda} \overline{\mathbf{H}}_{b}^{T}\left(\overline{\mathbf{H}}_{b} \boldsymbol{\Lambda} \overline{\mathbf{H}}_{b}^{T}\right)^{-1} \overline{\mathbf{H}}_{b} \boldsymbol{\Lambda} \overline{\mathbf{1}}
\end{aligned}
$$

We need to clarify that this residual demand derivative is the residual demand derivative for bus $n$ as a whole, i.e., the local actual demand at bus $n$ has been combined with the supply at the same bus. Therefore, from the point of view of the generation firm located at bus $n$, its residual demand derivative is actually (29) plus the local demand derivative at bus $n$, if there is any local demand.

For convenience in the above analysis, we calculate the residual demand derivative at the slack bus. Notice that (29) indicates that the residual demand derivative only depends on $\boldsymbol{\Lambda}$ and $\overline{\mathbf{H}}_{b}$, which are reduced matrices (with rows and/or columns corresponding to the slack bus deleted). For residual demand at an arbitrary bus $k$, all we need to do is to reconstruct $\boldsymbol{\Lambda}$ and $\overline{\mathbf{H}}_{b}$ assuming that bus $k$ is chosen as the slack bus in order to make use of (29).

\section{Weighted Least Squares Regression Interpretation}

The formula in (29) is the negative summed square error (SSE) of the following linear weighted least squares (WLS) regression problem: regress 1 on each column of $\overline{\mathbf{H}}_{b}$, and use $\boldsymbol{\Lambda}$ as the weight matrix. Suppose we have $n-1$ observations $\left(Y_{i}, \mathbf{X}_{i}\right), \forall i=1, \ldots, n-1$, where

$$
\begin{aligned}
{\left[\begin{array}{llll}
Y_{1} & Y_{2} & \ldots & Y_{n-1}
\end{array}\right]^{T}=\overline{\mathbf{1}} \equiv \mathbf{Y} } \\
{\left[\begin{array}{llll}
\mathbf{X}_{1} & \mathbf{X}_{2} & \ldots & \mathbf{X}_{n-1}
\end{array}\right]=\overline{\mathbf{H}}_{b} \equiv \mathbf{X}^{T} . }
\end{aligned}
$$

The linear WLS regression problem is to find an optimal $m \times 1$ vector $\boldsymbol{\beta}$ that minimizes the weighted sum of squared errors (see the Appendix for details)

$$
\min _{\boldsymbol{\beta}} S S E^{W L S}(\boldsymbol{\beta})=\sum_{i=1}^{n-1} w_{i}\left(Y_{i}-\mathbf{X}_{i}^{T} \boldsymbol{\beta}\right)^{2}
$$

where $w_{i}, i=1, \ldots, n$ satisfy

$$
\left[\begin{array}{cccc}
w_{1} & 0 & \ldots & 0 \\
0 & w_{2} & \ldots & 0 \\
\vdots & \vdots & \ddots & \vdots \\
0 & 0 & \ldots & w_{n-1}
\end{array}\right]=\mathbf{\Lambda} .
$$

The solution to this WLS problem is

$$
\begin{aligned}
\mathbf{b}^{W L S} & =\left(\overline{\mathbf{H}}_{b} \boldsymbol{\Lambda} \overline{\mathbf{H}}_{b}^{T}\right)^{-1} \overline{\mathbf{H}}_{b} \boldsymbol{\Lambda} \overline{\mathbf{1}}=\left|\frac{d \tilde{\boldsymbol{\mu}}}{d \lambda}(\hat{\lambda})\right|,(34) \\
S S E^{W L S}\left(\mathbf{b}^{W L S}\right) & =\overline{\mathbf{1}}^{T} \boldsymbol{\Lambda} \overline{\mathbf{1}}-\overline{\mathbf{1}}^{T} \boldsymbol{\Lambda} \overline{\mathbf{H}}_{b}^{T}\left(\overline{\mathbf{H}}_{b} \Lambda \overline{\mathbf{H}}_{b}^{T}\right)^{-1} \overline{\mathbf{H}}_{b} \Lambda \overline{\mathbf{1}} \\
& =\left|\frac{d \tilde{R}_{n}}{d \lambda}(\hat{\lambda})\right| .
\end{aligned}
$$

Such a least squares interpretation helps us gain insight into the original problem. (It is interesting that least squares interpretations are widely observed. Another example in the context of nodal prices can be found in [23], although the specific topic is somewhat different.)

From WLS theory (see the Appendix), we know that the WLS problem (32) could be transformed into an equivalent OLS problem. Define

$$
\begin{aligned}
& \mathbf{Y}^{*}=\Lambda^{1 / 2} \mathbf{Y} \\
& \mathbf{X}^{*}=\Lambda^{1 / 2} \mathbf{X}
\end{aligned}
$$

where

$$
\boldsymbol{\Lambda}^{1 / 2}=\left[\begin{array}{cccc}
w_{1}^{1 / 2} & 0 & \ldots & 0 \\
0 & w_{2}^{1 / 2} & \ldots & 0 \\
\vdots & \vdots & \ddots & \vdots \\
0 & 0 & \ldots & w_{n-1}^{1 / 2}
\end{array}\right]
$$

The equivalent OLS to (32) is

$$
\min _{\boldsymbol{\beta}} S S E^{O L S}(\boldsymbol{\beta})=\sum_{i=1}^{n-1}\left(Y_{i}^{*}-\mathbf{X}_{i}^{* T} \boldsymbol{\beta}\right)^{2} .
$$

The solution to this OLS (39) problem is exactly the same as the solution of the WLS problem (32)

$$
\begin{aligned}
\mathbf{b}^{O L S} & =\left(\overline{\mathbf{H}}_{b} \boldsymbol{\Lambda} \overline{\mathbf{H}}_{b}^{T}\right)^{-1} \overline{\mathbf{H}}_{b} \boldsymbol{\Lambda} \overline{\mathbf{1}}=\left|\frac{d \tilde{\boldsymbol{\mu}}}{d \lambda}(\hat{\lambda})\right| \\
S S E^{O L S}\left(\mathbf{b}^{O L S}\right) & =\overline{\mathbf{1}}^{T} \boldsymbol{\Lambda} \overline{\mathbf{1}}-\overline{\mathbf{1}}^{T} \boldsymbol{\Lambda} \overline{\mathbf{H}}_{b}^{T}\left(\overline{\mathbf{H}}_{b} \boldsymbol{\Lambda} \overline{\mathbf{H}}_{b}^{T}\right)^{-1} \overline{\mathbf{H}}_{b} \boldsymbol{\Lambda} \overline{\mathbf{1}} \\
& =\left|\frac{d \tilde{R}_{n}}{d \lambda}(\hat{\lambda})\right| .
\end{aligned}
$$


The residual of the OLS problem (39) with $\mathbf{b}^{O L S}$ specified in (40) is

$$
\mathbf{e}^{*}=\mathbf{Y}^{*}-\mathbf{X}^{*} \mathbf{b}^{O L S}=\boldsymbol{\Lambda}^{1 / 2}\left(\overline{\mathbf{1}}+\overline{\mathbf{H}}_{b}^{T} \frac{d \tilde{\boldsymbol{\mu}}}{d \lambda}(\hat{\lambda})\right) .
$$

From OLS theory (see the Appendix), we know $\mathbf{X}^{*}$ is orthogonal to $\mathbf{e}^{*}$, i.e.,

$$
\mathbf{X}^{* T} \mathbf{e}^{*}=\mathbf{0}
$$

i.e.,

$$
\overline{\mathbf{H}}_{b} \boldsymbol{\Lambda}\left(\overline{\mathbf{1}}+\overline{\mathbf{H}}_{b}^{T} \frac{d \tilde{\boldsymbol{\mu}}}{d \lambda}(\hat{\lambda})\right)=\mathbf{0}
$$

or

$$
\overline{\mathbf{H}}_{b} \boldsymbol{\Lambda}\left(\overline{\mathbf{1}}-\overline{\mathbf{H}}_{b}^{T}\left(\overline{\mathbf{H}}_{b} \boldsymbol{\Lambda} \overline{\mathbf{H}}_{b}^{T}\right)^{-1} \overline{\mathbf{H}}_{b} \boldsymbol{\Lambda} \overline{\mathbf{1}}\right)=\mathbf{0}
$$

by substituting $(d \tilde{\mu} / d \lambda)(\hat{\lambda})$ from (26). Note that (45) is the same as (27).

The orthogonality condition (43) produces the same equation as (25), so we would like to call (25) the orthogonality equation of binding constraints. The meaning of (25) is that when binding constraints do not change, the Jacobian of the constraints $\left(\overline{\mathbf{H}}_{b}\right.$ in this case) is orthogonal to the direction of change $(d \tilde{\overline{\mathbf{q}}} / d \lambda)(\hat{\lambda})$. In other words, the direction of change $(d \overline{\overline{\mathbf{q}}} / d \lambda)(\hat{\lambda})$ will not change these binding constraints: no active constraints will be violated, and no active constraints will become nonbinding. It is the orthogonality equation of binding constraints that makes possible the WLS interpretation.

In addition, from WLS theory (see the Appendix), we can deduce the following properties about the residual demand derivative.

1) Property 1: If $\boldsymbol{\Lambda}$ is positive definite, then the residual demand derivative is less than or equal to zero.

2) Property 2: Enforcing a new linearly independent binding constraint in the OPF will reduce the residual demand derivative in absolute value if

$$
\mathbf{M}_{\mathbf{X}^{*}}\left(\bar{H}_{b}^{\text {Added }}\right)^{T} \neq 0
$$

and

$$
\mathbf{M}_{\mathbf{X} * \overline{\mathbf{1}}^{T}} \neq 0
$$

where $\mathbf{X}^{*}=\Lambda^{1 / 2} \overline{\mathbf{H}}_{b}^{T}$ as defined in (37)

$$
\mathbf{M}_{\mathbf{X}^{*}}=\overline{\mathbf{I}}-\mathbf{X}^{*}\left(\mathbf{X}^{* T} \mathbf{X}^{*}\right)^{-1} \mathbf{X}^{* T}
$$

and $\bar{H}_{b}^{\text {Added }}$ is an added row to $\overline{\mathbf{H}}_{b}$.
In particular, if there is no transmission congestion, the residual demand derivative is $-\overline{\mathbf{1}}^{T} \boldsymbol{\Lambda} \overline{\mathbf{1}}$. When transmission is congested, the residual demand derivative decreases in absolute value, because

$$
\left|\frac{d R_{n}}{d \lambda}(\hat{\lambda})\right|=S S E_{C}^{*} \leq \overline{\mathbf{1}}^{T} \boldsymbol{\Lambda} \overline{\mathbf{1}}=S S E_{N C}^{*}
$$

where the subscript " $C$ " denotes "transmission congested," and the subscript " $N C$ " denotes "transmission uncongested." This implies that when transmission constraints bind, the players have more incentive to exert market power because of the decrease in magnitude of the residual demand derivative.

We have assumed that there is no perfectly elastic supply at any bus in the derivation of (29). If there is perfectly elastic supply at some bus, then (29) is not valid, because we cannot invert a singular matrix to get $\boldsymbol{\Lambda}$ in (28). To consider perfectly elastic supply, we will analyze the limit of the residual demand derivative as some diagonal elements of $\boldsymbol{\Lambda}$ go to infinity. We are especially interested in the conditions under which the residual demand derivative goes to infinity, i.e., the residual demand is perfectly elastic.

Theorem 1: Suppose there are $l$ buses $\left(i_{1}, i_{2}, \ldots, i_{l}\right)$, each with its supply derivative going to infinity, and denote the set composed of $i_{1}, i_{2}, \ldots, i_{l}$ as $\mathbb{L}$. If the following (50) has solution, then the residual demand derivative at the slack bus is bounded; otherwise, the residual demand derivative at the slack bus goes to infinity:

$$
\left[\begin{array}{c}
\mathbf{X}_{i_{1}}^{T} \\
\mathbf{X}_{i_{2}}^{T} \\
\vdots \\
\mathbf{X}_{i_{l}}^{T}
\end{array}\right] \boldsymbol{\beta}=\mathbf{1}_{l}
$$

where $\mathbf{1}_{l}=\underbrace{\left[\begin{array}{llll}1 & 1 & \ldots & 1\end{array}\right]^{T}}_{l}$.

Proof:

1) Suppose (50) has a solution $\hat{\boldsymbol{\beta}}$. From (32), we have

$\min _{\boldsymbol{\beta}} S S E^{W L S}(\boldsymbol{\beta})$

$$
\leq S S E^{W L S}(\hat{\boldsymbol{\beta}})=\sum_{i=1, i \notin \mathbb{L}}^{n-1} w_{i}\left(1-\mathbf{X}_{i}^{T} \hat{\boldsymbol{\beta}}\right)^{2} .
$$

Therefore, if (50) has solution, the residual demand derivative at the slack bus is bounded.

2) Suppose (50) does not have a solution. Choose any $\tilde{\boldsymbol{\beta}}$ that satisfies

$$
\tilde{\boldsymbol{\beta}} \in \arg \min _{\boldsymbol{\beta}} \sum_{i \in \mathbb{L}}\left(1-\mathbf{X}_{i}^{T} \boldsymbol{\beta}\right)^{2} .
$$

By assumption

$$
\sum_{i \in \mathbb{L}}\left(1-\mathbf{X}_{i}^{T} \tilde{\boldsymbol{\beta}}\right)^{2} \neq 0
$$


From (32), we have

$$
\begin{aligned}
& \min _{\boldsymbol{\beta}} S S E^{W L S}(\boldsymbol{\beta}) \\
& =\min _{\boldsymbol{\beta}} \sum_{i=1}^{n-1} w_{i}\left(Y_{i}-\mathbf{X}_{i}^{T} \boldsymbol{\beta}\right)^{2} \\
& \geq \min _{\boldsymbol{\beta}} \sum_{i \in \mathbb{L}} w_{i}\left(1-\mathbf{X}_{i}^{T} \boldsymbol{\beta}\right)^{2} \\
& \geq \min _{\boldsymbol{\beta}} \sum_{i \in \mathbb{L}}\left(\min _{k \in \mathbb{L}} w_{k}\right)\left(1-\mathbf{X}_{i}^{T} \boldsymbol{\beta}\right)^{2} \\
& =\left(\min _{k \in \mathbb{L}} w_{k}\right)\left(\min _{\boldsymbol{\beta}} \sum_{i \in \mathbb{L}}\left(1-\mathbf{X}_{i}^{T} \boldsymbol{\beta}\right)^{2}\right) .
\end{aligned}
$$

By the definition of $\tilde{\boldsymbol{\beta}}$

$$
\min _{\boldsymbol{\beta}} S S E^{W L S}(\boldsymbol{\beta}) \geq\left(\min _{k \in \mathbb{L}} w_{k}\right) \sum_{i \in \mathbb{L}}\left(1-\mathbf{X}_{i}^{T} \tilde{\boldsymbol{\beta}}\right)^{2} .
$$

Therefore

$$
\begin{aligned}
& \lim _{w_{i} \rightarrow \infty, \forall i \in \mathbb{L}}\left(\min _{\boldsymbol{\beta}} S S E^{W L S}(\boldsymbol{\beta})\right) \\
\geq & \lim _{w_{i} \rightarrow \infty, \forall i \in \mathbb{L}}\left(\left(\min _{k \in \mathbb{L}} w_{k}\right) \sum_{i \in \mathbb{L}}\left(1-\mathbf{X}_{i}^{T} \tilde{\boldsymbol{\beta}}\right)^{2}\right)=\infty .
\end{aligned}
$$

Therefore, if (50) does not have solution, the residual demand derivative at the slack bus is unbounded.

Generally speaking, if the number of buses with perfectly elastic supply is greater than the number of binding constraints, then the residual demand derivative at the slack bus is unbounded, because there are more equations than variables in (50), unless enough number of equations in (50) are redundant.

When the residual demand derivative is bounded, it could be calculated from the WLS problem (32). Essentially, all buses that have perfectly elastic supply must have zero residual

$$
e_{i}^{*}=0, \quad \forall i \in \mathbb{L}
$$

in order to zero out the arbitrarily large $w_{i}, \forall i \in \mathbb{L}$. We will show an example for this case in Section III-C.

The WLS interpretation and the fact (55) have an important implication. Increasing the quantity-price response makes the electricity market more competitive. As shown in (33), larger quantity-price response will have a larger weight in the WLS problem. However, increased quantity-price response is not effective if binding transmission constraints prevent the large quantity-price responses from "spreading out" to the whole system. Our analysis allows the determination of the quantity-price response in the presence of transmission constraints.

\section{E. Non-Differentiable Case}

The OPF solution might be at a point of non-differentiability. In other words, the sensitivity with respect to the slack bus price

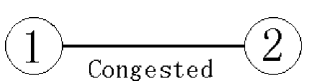

Fig. 1. Two-bus example.

is not defined. This occurs when there are just binding constraints and/or the current OPF solution is at a point of non-differentiability of a supply function. If we encounter the case where the left-side and right-side residual demand derivative exist, but they are not equal, we could calculate the left-side and right-side residual demand derivative, respectively. If we can determine which constraints are binding as the price at bus $n$ increases or decreases, then the left-side and right-side residual demand derivatives are specified by (29) or can be calculated by the equivalent WLS problem for perfectly elastic supply/demand case with the corresponding binding constraints. Determining the binding constraints in general involves enumerating each possible combination of binding constraints and, for each, checking if the solution implied by sensitivity analysis will indeed induce the same set of binding constraints. Similar analysis is also necessary in the case that the OPF solution is at a kink point of a supply function; that is, the left-side derivative does not equal the right-side derivative.

\section{EXAMPLES}

\section{A. Example 1: Intuitive Two-Bus Case}

Consider the simplest case of a two-bus system connected by a single transmission line, and the line is congested. The case is illustrated in Fig. 1.

Many researchers have adopted this example to illustrate the transmission effect on the equilibrium. (For example, see Borenstein et al. [22].) It is observed that the market equilibrium just resembles the combination of two decoupled single bus system equlibria. We will demonstrate that the analysis in Section II is consistent with this observation. We compute the residual demand at bus 2 and choose it as the slack bus. Because $m_{b}=1$ and $n=2, \overline{\mathbf{H}}_{b}$ is a $1 \times 1$ matrix, and $\overline{\mathbf{H}}_{b}=1$. Substitute $\overline{\mathbf{H}}_{b}=1$ into (26) and (27), and we get $(d \mu / d \lambda)(\hat{\lambda})=-1$ and $\left(d q_{1} / d \lambda\right)(\hat{\lambda})=0$, which implies that the supply at bus 1 is not affected by the price at bus 2 . Also substitute $\overline{\mathbf{H}}_{b}=1$ into (29), and we get $\left(d R_{2} / d \lambda\right)(\hat{\lambda})=0$. Since we have combined the supply and demand at bus 2 , so this implies that the residual demand at bus 2 is just the derivative of the actual local demand derivative at bus 2 , and the market at bus 1 does not affect the residual demand derivative at bus 2 . Similar results hold for bus 1 . These results verify the intuitive result that the market is decoupled in this case.

\section{B. Example 2: Numerical Four-Bus Case}

This example is illustrated in Fig. 2. We consider a two-loop system from [5]. Each branch admittance equals 0.1. There are two generation firms located at bus 1 and 2 and two loads located at bus 3 and 4 . 


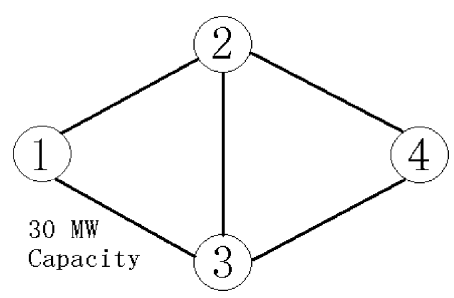

Fig. 2. Four-bus example.

The marginal generation cost functions and demand functions in bus order are

$$
\begin{array}{ll}
\text { Generation } & \text { Load } \\
p_{G 1}=10+0.35 q_{1} & D_{L 3}=375-1.92 p_{3} \\
p_{G 2}=10+0.45 q_{2} & D_{L 4}=225-1.94 p_{4} .
\end{array}
$$

Branch 1-3 has a transmission capacity limit of $30 \mathrm{MW}$, and all the other branches have transmission capacity limits of $400 \mathrm{MW}$. Following $\mathrm{Xu}$ and $\mathrm{Yu}$, we consider the existence of a transmission-constrained linear SFE for this case. The transmission-constrained linear SFE

$$
S_{i}\left(p_{i}\right)=\beta_{i}\left(p-\alpha_{i}\right), i=1, \ldots, n
$$

is characterized by [5]

$$
\begin{aligned}
\frac{\beta_{i}^{*}}{1-c_{i} \beta_{i}^{*}} & =-R_{i}^{* \prime} \\
\alpha_{i}^{*} & =e_{i}
\end{aligned}
$$

where generation firm $i$ 's linear marginal cost function is $C_{i}^{\prime}\left(q_{i}\right)=c_{i} q_{i}+e_{i}$.

Now we demonstrate that our analysis confirms to the numerical results in [5]. The way we verify it is as follows.

A linear supply function best response could be calculated directly from a generation firm's profit maximization problem as $\mathrm{Xu}$ and $\mathrm{Yu}$ did in [5]. Because the supply function best response and the residual demand derivative satisfy (1), we could solve for the residual demand derivative with a given supply function best response. The solution is (56). We used $\mathrm{Xu}$ and Yu's numerical supply function best response solutions as input, and we solved for numerical residual demand derivatives using (56). Recall that we have derived the analytical solution of the residual demand derivative in (29). Therefore, we could compare our analytic solutions derived in this paper with the numerical solutions recovered from $\mathrm{Xu}$ and Yu's results using (56).

In particular, denote the output of the algorithm in [5] by $\hat{\boldsymbol{\beta}}$, which is the input to recover $R_{i}^{* \prime}$ using (29), and denote the so-recovered $R_{i}^{* \prime}$ by $\hat{R}_{i}$ '. Then we calculate analytically the $R_{i}^{* \prime}$ values evaluated at $\hat{\beta}$ and compare $R_{i}^{* \prime}$ calculated analytically to $\hat{R}_{i}^{\prime}$ from the output of the algorithm in [5]. The results are summarized in Table I.

Define the relative numerical error by

$$
R E_{i}=\frac{\left|R_{i}^{* \prime}-\hat{R}_{i}^{\prime}\right|}{\left|R_{i}^{* \prime}\right|} \times 100 \% .
$$

The smaller the $R E_{i}$, the more closely our results conform with $\mathrm{Xu}$ and Yu's results.
TABLE I

RESULTS COMPARISON

\begin{tabular}{lll}
\hline \hline Tol $=0.01$ & Bus 1 & Bus 2 \\
\hline$\hat{\beta}_{i}$ & $1 / 7.803$ & $1 / 1.256$ \\
\hline$\hat{R}_{i}^{\prime}$ & -0.1342 & -1.2407 \\
\hline$R_{i}^{* \prime}$ & -0.1290 & -1.1824 \\
\hline$R E_{i}$ & $4 \%$ & $5 \%$ \\
\hline \hline Tol $=0.001$ & Bus 1 & Bus 2 \\
\hline$\hat{\beta}_{i}$ & $1 / 8.343$ & $1 / 1.329$ \\
\hline$\hat{R}_{i}^{\prime}$ & -0.1251 & -1.1377 \\
\hline$R_{i}^{* \prime}$ & -0.1246 & -1.1325 \\
\hline$R E_{i}$ & $0.4 \%$ & $0.5 \%$ \\
\hline \hline Tol $=0.0001$ & Bus 1 & Bus 2 \\
\hline$\hat{\beta}_{i}$ & $1 / 8.399$ & $1 / 1.337$ \\
\hline$\hat{R}_{i}^{\prime}$ & -0.1242 & -1.1274 \\
\hline$R_{i}^{* \prime}$ & -0.1241 & -1.1276 \\
\hline$R E_{i}$ & $0.08 \%$ & $0.02 \%$ \\
\hline \hline
\end{tabular}

Table I shows that the relative error is in the range of $5 \%$ to $0.02 \%$. Moreover, as the numerical accuracy of $\mathrm{Xu}$ and Yu's results increases as specified by a tolerance parameter in the stopping criterion of Xu and Yu's algorithm, $R E_{i}$ decreases, which indicates a better confirmation. The parameter $T o l$ is the tolerance control parameter in [5], which controls the output accuracy of the bidding parameter $\hat{\boldsymbol{\beta}}$. Note that smaller $T o l$ results in smaller $R E_{i}$.

From the results, we conclude that the characterization in this paper is consistent with the numerical results in [5].

\section{Example 3: Numerical Three-Bus Case With Perfectly Elastic Supply}

This example is taken from [1], and we simplify it by only considering information that is relevant to the residual demand derivative calculation. As illustrated in Fig. 3, it is a three-bus system with line 2-3 having a capacity of $600 \mathrm{MW}$. All other lines have very large capacity and cannot be congested. All three lines have the same impedance. There are three generators G1, G2, and G3 located at the corresponding buses. G1 bids a linear supply function with slope

$$
S_{1}^{\prime}(p)=1000 \frac{\mathrm{MW}}{\left(\frac{\mathrm{cent}}{\mathrm{kWh}}\right)}
$$

and G3 has perfectly elastic supply. There is only one load, which is located at bus 3 .

We want to calculate the residual demand derivative at bus 2 , so we designate bus 2 as the slack bus. Since there is a perfectly elastic supply at bus 3 , we can use the WLS interpretation technique to calculate the residual demand derivative as the limit as a weight approaches infinity. We have for this case

$$
\overline{\mathbf{H}}_{b}=\left[\begin{array}{ll}
-\frac{1}{3} & -\frac{2}{3}
\end{array}\right]
$$




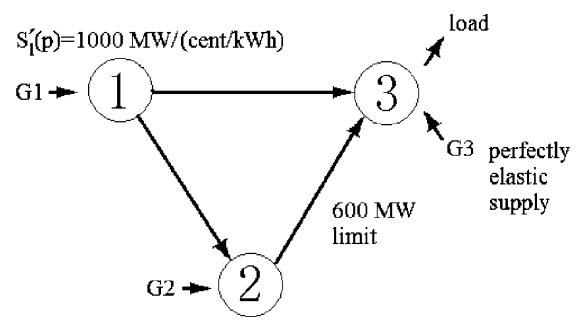

Fig. 3. Three-bus example with perfectly elastic supply.

and

$$
\boldsymbol{\Lambda}=\left[\begin{array}{cc}
1000 & 0 \\
0 & W_{2}
\end{array}\right]
$$

where $W_{2}$ denotes an arbitrarily large number. Form the WLS problem as follows:

$$
\min _{\beta} S S E^{W L S}(\beta)=1000\left(1+\frac{1}{3} \beta\right)^{2}+M\left(1+\frac{2}{3} \beta\right)^{2} \text {. }
$$

The solution of this problem is

$$
b^{W L S}=\frac{-2000-2 W_{2}}{\frac{2000}{3}+\frac{4 W_{2}}{3}} .
$$

Take the limit of $b^{W L S}$ as $W_{2} \rightarrow \infty$, and we have

$$
\lim _{W_{2} \rightarrow \infty} b^{W L S}=\lim _{W_{2} \rightarrow \infty} \frac{-2000-2 W_{2}}{\frac{2000}{3}+\frac{4 W_{2}}{3}}=-\frac{3}{2} .
$$

Therefore

$$
\begin{aligned}
\lim _{W_{2} \rightarrow \infty}\left(\min _{\beta} S S E^{W L S}(\beta)\right) & =\lim _{W_{2} \rightarrow \infty}\left(S S E^{W L S}\left(b^{W L S}\right)\right) \\
& =1000\left(1+\frac{1}{3}\left(-\frac{3}{2}\right)\right)^{2}=250 .
\end{aligned}
$$

Therefore, the residual demand derivative at bus 2 is -250 . Notice that the optimal $b^{W L S}$ makes

$$
1+\frac{2}{3} b^{W L S}=0 .
$$

Generally, the optimal WLS coefficients should make the residual, corresponding to a bus with perfectly elastic supply, equal to zero. Otherwise, the WLS problem will be unbounded.

In [1], Cardell et al. characterized the inverse of the residual demand derivative at bus 2 in (2). Their calculation is based on their intuitive price relationship

$$
p_{2}=2 p_{1}-p_{3} .
$$

They obtained the solution -0.004 for the inverse residual demand derivative as indicated in [1, equation (4)]. Therefore, our calculation is consistent with this solution, because

$$
\frac{1}{-0.004}=-250 \text {. }
$$

\section{CONCLUSION}

We characterize the residual demand at the slack bus based on the FONCs of the OPF problem. The residual demand curve is implicitly characterized by eliminating the equations in the FONCs that contain the offer information of the generation firm located at the slack bus. After doing that, there is one degree of freedom left in the FONCs that defines a locus of $\left(\lambda, q_{n}\right)$, i.e., the residual demand curve. We obtain the residual demand derivative formula (29) by sensitivity analysis viewing the price at the slack bus as a parameter and assuming there is no perfectly elastic supply in the system. The solution has a suggestive WLS interpretation. Several useful properties of the residual demand derivative are implied by WLS theory. If there is perfectly elastic supply at some bus in the system, it is convenient to use the WLS interpretation to analyze the limit of the residual demand derivative as the quantity-price response of the bus goes to infinity. We establish the condition under which the residual demand derivative at another bus will be bounded or unbounded in this case. All the residual demand derivative analysis is applicable to both the supply function model and the Cournot model.

The correctness of the residual demand derivative analytic formulation is verified using an intuitive two-bus system, a numerical four-bus two-loop system from [5], and a three-bus oneloop system from [1].

The residual demand derivative formulation could be used to construct optimal bidding strategies in a transmission-constrained network, and it could be incorporated into sophisticated optimal strategy and market equilibrium algorithms. It also provides useful information and insights for market analysts and market monitoring entities.

\section{APPENDIX \\ ORDINARY LEAST SQUARES PROBLEM AND Weighted Least SQuares PROBlem}

The formulation and results in this section are from [24]. An OLS problem is formulated as follows. Suppose there are $n$ observations $\left(Y_{i}, \mathbf{X}_{i}\right), \forall i=1, \ldots, n$. The objective is to find an optimal vector $\boldsymbol{\beta}$ that minimizes the SSE

$$
\min _{\boldsymbol{\beta}} S S E^{O L S}(\boldsymbol{\beta})=\sum_{i=1}^{n}\left(Y_{i}-\mathbf{X}_{i}^{T} \boldsymbol{\beta}\right)^{2} .
$$

The solution to this OLS problem is

$$
\mathbf{b}^{O L S}=\left(\mathbf{X}^{T} \mathbf{X}\right)^{-1} \mathbf{X}^{T} \mathbf{Y}
$$

where

$$
\begin{aligned}
& \mathbf{X}=\left[\begin{array}{c}
\mathbf{X}_{1}^{T} \\
\mathbf{X}_{2}^{T} \\
\vdots \\
\mathbf{X}_{n}^{T}
\end{array}\right] \\
& \mathbf{Y}=\left[\begin{array}{llll}
Y_{1} & Y_{2} & \ldots & Y_{n}
\end{array}\right]^{T}
\end{aligned}
$$

assuming there is no multicollinearity, i.e., $\mathbf{X}$ has linearly independent columns. 
The minimal SSE is

$$
S S E^{O L S}\left(\mathbf{b}^{O L S}\right)=\mathbf{Y}^{T}\left(\mathbf{I}-\mathbf{X}\left(\mathbf{X}^{T} \mathbf{X}\right)^{-1} \mathbf{X}^{T}\right) \mathbf{Y}
$$

Define a projection matrix $\mathbf{P}_{\mathbf{X}}$ by

$$
\mathbf{P}_{\mathbf{X}}=\mathbf{X}\left(\mathbf{X}^{T} \mathbf{X}\right)^{-1} \mathbf{X}^{T}
$$

Define another projection matrix $\mathbf{M}$ by

$$
\mathbf{M}_{\mathbf{X}}=\mathbf{I}-\mathbf{P}_{\mathbf{X}}
$$

Both matrices $\mathbf{P}_{\mathbf{X}}$ and $\mathbf{M}_{\mathbf{x}}$ are idempotent, namely

$$
\begin{aligned}
\mathbf{P}_{\mathbf{X}}^{2} & =\mathbf{P}_{\mathbf{X}} \\
\mathbf{M}_{\mathbf{X}}^{2} & =\mathbf{M}_{\mathbf{X}} .
\end{aligned}
$$

In addition, both matrices $\mathbf{P}$ and $\mathbf{M}$ are positive semi-definite so that

$$
S S E^{O L S}\left(\mathbf{b}^{O L S}\right)=\mathbf{Y}^{T} \mathbf{M}_{\mathbf{X}} \mathbf{Y} \geq 0 .
$$

Define the residual

$$
\mathbf{e}=\mathbf{M}_{\mathbf{X}} \mathbf{Y}
$$

then

$$
\mathbf{X}^{T} \mathbf{e}=\mathbf{0}
$$

Suppose we want to add one regressor to the problem. Now there are $n$ observations $\left(Y_{i}, \mathbf{X}_{i}, z_{i}\right), \forall i=1, \ldots, n$, with $z_{i}$ added. Again assume there is no multicollinearity with $z_{i}$ added.

The objective is to find an optimal vector $\boldsymbol{\beta}$ and $\gamma$ that minimizes the SSE

$$
\min _{\boldsymbol{\beta}, \boldsymbol{\gamma}} S S E^{O L S}(\boldsymbol{\beta}, \gamma)=\sum_{i=1}^{n}\left(Y_{i}-\mathbf{X}_{i}^{T} \boldsymbol{\beta}-z_{i} \gamma\right)^{2} .
$$

We have

$$
\begin{aligned}
\left\{\min _{\boldsymbol{\beta}, \boldsymbol{\gamma}} S S E^{O L S}(\boldsymbol{\beta}, \gamma)\right\} \\
=\left\{\min _{\boldsymbol{\beta}} S S E^{O L S}(\boldsymbol{\beta})\right\}-c^{2}\left(\mathbf{z}^{T} \mathbf{M}_{\mathbf{X} \mathbf{z}}\right)
\end{aligned}
$$

where $\mathbf{z}=\left[\begin{array}{llll}z_{1} & z_{2} & \ldots & z_{n}\end{array}\right]^{T}$, and

$$
c=\left(\mathbf{z}^{T} \mathbf{M}_{\mathbf{X}} \mathbf{z}\right)^{-1} \mathbf{z}^{T} \mathbf{M}_{\mathbf{X}} \mathbf{Y} \text {. }
$$

Note that if

$$
\mathbf{M}_{\mathbf{X}} \mathbf{z} \neq 0
$$

and

$$
\mathbf{M}_{\mathbf{X}} \mathbf{Y} \neq 0
$$

then

$$
\min _{\boldsymbol{\beta}, \boldsymbol{\gamma}} S S E^{O L S}(\boldsymbol{\beta}, \gamma)<\min _{\boldsymbol{\beta}} S S E^{O L S}(\boldsymbol{\beta})
$$

If we put weights on different observations, then it is a WLS problem with the following objective:

$$
\min _{\boldsymbol{\beta}} S S E=\sum_{i=1}^{n} w_{i}\left(Y_{i}-\mathbf{X}_{i}^{T} \boldsymbol{\beta}\right)^{2} .
$$

The solution to this WLS problem is

$$
\mathbf{b}^{W L S}=\left(\mathbf{X}^{T} \mathbf{W X}\right)^{-1} \mathbf{X}^{T} \mathbf{W} \mathbf{Y}
$$

where the weight matrix is defined by

$$
\mathbf{W}=\left[\begin{array}{cccc}
W_{1} & 0 & \ldots & 0 \\
0 & W_{2} & \ldots & 0 \\
\vdots & \vdots & \ddots & \vdots \\
0 & 0 & \ldots & W_{n}
\end{array}\right]
$$

The minimal SSE is

$S S E^{W L S}\left(\mathbf{b}^{W L S}\right)=\mathbf{Y}^{T}\left(\mathbf{W}-\mathbf{X}\left(\mathbf{X}^{T} \mathbf{W X}\right)^{-1} \mathbf{X}^{T}\right) \mathbf{Y}$

Let us assume $\mathbf{W}$ is positive semi-definite so that $\mathbf{W}^{1 / 2}$ exists

$$
\mathbf{W}^{1 / 2} \mathbf{W}^{1 / 2}=\mathbf{W} \text {. }
$$

The WLS could be transformed to an equivalent OLS problem by defining

$$
\begin{aligned}
& \mathbf{Y}^{*}=\mathbf{W}^{1 / 2} \mathbf{Y} \\
& \mathbf{X}^{*}=\mathbf{W}^{1 / 2} \mathbf{X}
\end{aligned}
$$

where

$$
\mathbf{W}^{1 / 2}=\left[\begin{array}{cccc}
w_{1}^{1 / 2} & 0 & \ldots & 0 \\
0 & w_{2}^{1 / 2} & \ldots & 0 \\
\vdots & \vdots & \ddots & \vdots \\
0 & 0 & \ldots & w_{n-1}^{1 / 2}
\end{array}\right]
$$

The equivalent OLS to (32) is

$$
\min _{\boldsymbol{\beta}} S S E^{O L S}(\boldsymbol{\beta})=\sum_{i=1}^{n-1}\left(Y_{i}^{*}-\mathbf{X}_{i}^{* T} \boldsymbol{\beta}\right)^{2} .
$$

\section{REFERENCES}

[1] J. B. Cardell, C. C. Hitt, and W. W. Hogan, "Market power and strategic interaction in electricity networks," Res. Energy Econ., vol. 19, pp. 109-137, 1997.

[2] J. Yao, S. S. Oren, and I. Adler, "Computing Cournot equilibria in twosettlement electricity markets with Transmission constraints," in Proc. 37th Hawaii Int. Conf. Systems Sciences (HICCS 37), Big Island, HI, 2004.

[3] C. Berry, B. Hobbs, W. Meroney, R. O’Neill, and W. Stewart, "Understanding how market power can arise in network competition: A game theoretic approach," Util. Pol., vol. 8, pp. 139-158, 1999.

[4] B. F. Hobbs, C. B. Metzler, and J.-S. Pang, "Strategic gaming analysis for electric power systems: An MPEC approach," IEEE Trans. Power Syst., vol. 15, no. 2, pp. 638-645, May 2000.

[5] L. Xu and Y. Yu, "Transmission constrained linear supply function equilibrium in power markets: Method and example," in Proc. Int. Conf. Power System Technology, Kunming, China, Oct. 2002, vol. 3, pp. 1349-1354. 
[6] S. Stoft, Power System Economics. Piscataway, NJ: IEEE Press, 2002.

[7] P. D. Klemperer and M. A. Meyer, "Supply function equilibria in oligopoly under uncertainty," Econometrica, vol. 57, no. 6, pp. 1243-1277, Nov. 1989.

[8] R. J. Green and D. M. Newbery, "Competition in the British electricity spot market," J. Polit. Econ., vol. 100, no. 5, pp. 929-953, Oct. 1992.

[9] R. Green, "Increasing competition in the British electricity spot market," J. Ind. Econ., vol. 44, no. 2, pp. 205-216, Jun. 1996.

[10] A. Rudkevich, "Supply Function Equilibrium in Power Markets: Learning All the Way," TCA Tech. Rep., Tabors Caramanis and Associates, 1999.

[11] R. Baldick, R. Grant, and E. Kahn, "Theory and application of linear supply function equilibrium in electricity markets," J. Reg. Econ., vol. 25, no. 2, pp. 143-167, 2004.

[12] R. Baldick and W. Hogan, Capacity Constrained Supply Function Equilibrium Models for Electricity Markets: Stability, Non-Decreasing Constraints, and Function Space Iterations, University of California Energy Institute, 2002, POWER Paper PWP-089.

[13] P. Holmberg, Unique Supply Function Equilibrium with Capacity Constraints, Uppsala University, Department of Economics, Working Paper 2004:20.

[14] P. Holmberg, Asymmetric Supply Function Equilibrium with Capacity Constraints, Uppsala University, Department of Economics, Working Paper 2005:12.

[15] E. J. Anderson and H. Xu, "Supply function equilibrium in electricity spot markets with contracts and prices caps," J. Optim. Theory Appl., vol. 60, pp. 215-283, Feb. 2005.

[16] T. Genc and S. Reynolds, Supply Function Equilibria with Pivotal Electricity Suppliers, Univ. Arizona, 2004, Eller College Working Paper-04.

[17] A. Hortaçsu and S. L. Puller, Testing Strategic Model of Firm Behavior in Restructured Electricity Markets: A Case Study of ERCOT, University of California Energy Institute, 2004, CSEM Working Paper 125.

[18] E. J. Anderson and A. B. Philpott, "Using supply functions for offering generation into an electricity market," Oper. Res., vol. 50, no. 3, pp. 477-489, 2002.
[19] C. E. Murillo-Sanchez, S. M. Ede, T. D. Mount, R. J. Thomas, and R. D. Zimmerman, "An engineering approach to monitoring market power in restructured markets for electricity," in Proc. 24th Annu. Int. Conf., Houston, TX, Apr. 2001.

[20] B. C. Lesieutre, H. S. Oh, R. J. Thomas, and V. Donde, "Identification of market power in large-scale electric energy markets," in Proc. 39th Hawaii Int. Conf. System Sciences, HI, 2006.

[21] H. Singh, S. Hao, and A. Papalexopoulos, "Transmission congestion management in competitive electricity markets," IEEE Trans. Power Syst., vol. 13, no. 2, pp. 672-680, May 1998.

[22] S. Borenstein, J. Bushnell, and S. Stoft, "The competitive effects of transmission capacity in a deregulated electricity industry," RAND J. Econ., vol. 31, pp. 294-325.

[23] T. Orfanogianni and G. Gross, "A general formulation for LMP evaluation,” IEEE Trans. Power Syst., vol. 22, no. 3, pp. 1163-1173, Aug. 2007.

[24] W. H. Greene, Econometric Analysis, 6 ed. Upper Saddle River, NJ: Prentice-Hall, 2000.

Lin Xu (S'07) received the B.S. degree and the M.E. degree in electrical engineering from Tianjin University, Tianjin, China, in 2000 and 2003, respectively. $\mathrm{He}$ is currently pursuing the Ph.D. degree in the Department of Electrical and Computer Engineering at The University of Texas at Austin.

Ross Baldick (F'07) received the B.Sc. degree in mathematics and physics and the B.E. degree in electrical engineering from the University of Sydney, Sydney, Australia, and the M.S. and Ph.D. degrees in electrical engineering and computer sciences in 1988 and 1990, respectively, from the University of California, Berkeley.

From 1991 to 1992, he was a Postdoctoral Fellow at the Lawrence Berkeley Laboratory. In 1992 and 1993, he was an Assistant Professor at Worcester Polytechnic Institute, Worcester, MA. He is currently a Professor in the Department of Electrical and Computer Engineering at The University of Texas at Austin. 